\title{
EDITORIAL
}

\author{
Jörg Schröder · Doru C. Lupascu
}

\section{Advanced modeling of ferroic materials}

Received: 6 April 2019 / Accepted: 7 April 2019 / Published online: 15 April 2019

(C) Springer-Verlag GmbH Germany, part of Springer Nature 2019

Ferroic materials play an important role in many modern technical devices ranging from consumer market items to applications in information technologies, high-end equipment for automotive, aircraft and spacecraft, and medical technology. Permanent magnets are the best-known ferroic material systems. Their magnetic ordering is due to the ferromagnetic phase transition. Permanent magnets are then typically stabilized by defects to avoid demagnetization during use. The multitude of electrical motors, transformers, and most other inductors accompany our daily life. They rely on these magnetic materials. Different from the magnetic systems, ferroelectric materials exhibit local electric order. Their largest market resides in electrical capacitors due to the large associated dielectric constant. The second largest market is piezoelectrics yielding a coupling between electrical and mechanical fields. Ferroelastic materials have recently gained increased interest in the context of smart structures including medical applications. Shape memory alloys are presently their most investigated subclass.

Among the functional materials, smart materials transform one fundamental physical property into another, namely mechanical to electric, magnetic to electric, or magnetic to mechanical fields. The development of devices utilizing smart materials, as well as their testing, is typically expensive. Therefore, considerable effort has been made to develop modeling tools that allow bypassing many of the experimental steps previously required in the design.

In order to capture the complexity of these materials effects, multiple scales have to be considered. Within this special issue, we have collected a number of contributions that focus on advanced modeling techniques of ferroic materials on different scales. The scientific contributions are the result of the efforts of the research groups united in the DFG Research Unit 1509 "Ferroic Functional Materials-Multiscale Modeling and Experimental Characterization" financed by the German research foundation. We have been able to span modeling from ab initio to common macroscopic finite element approaches. Phase field models, as well as multiscale homogenization methods, bridge these scale differences. We hope the readership will enjoy this set of works.

Publisher's Note Springer Nature remains neutral with regard to jurisdictional claims in published maps and institutional affiliations. 